\title{
OPTICAL DISK : REVIEW AND ANALYSIS
}

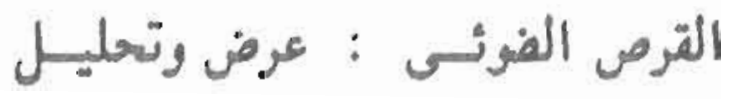

Prof. Dr. Ali I. EI-Desouky

Ass. Prof. Dr. Mofreh M. Salem"
Prof. Dr. Nadia H. Hegazi ${ }^{\text {** }}$ Eng. Ola A. Zahran ${ }^{* *}$

* Computers \& Control Eng. Dept., Faculty of Eng., El-Mansoura Uni., Egypt.

** Computers Dept., Electronics Research Institute, Doki, Cairo, Egypt.

Suresil.

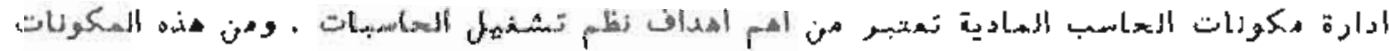

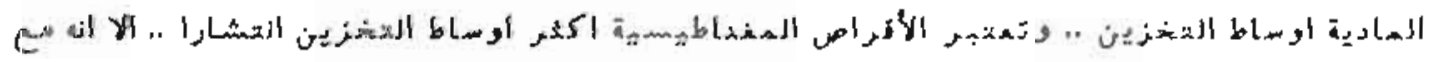

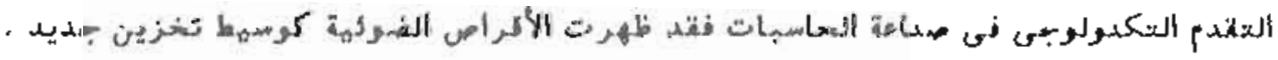

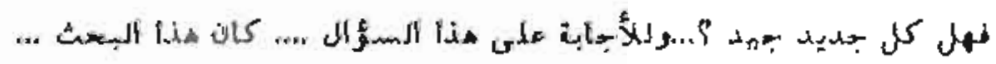

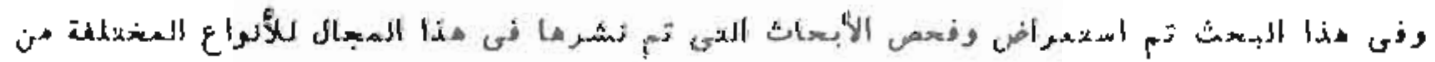

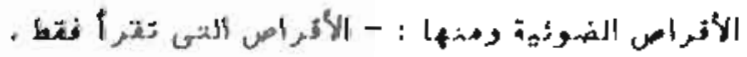

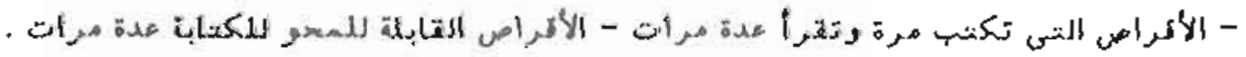
ولمالمسرو

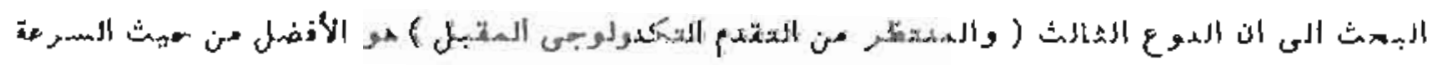

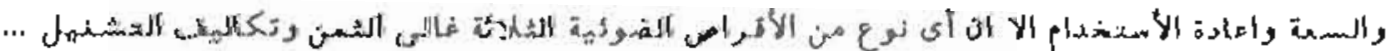

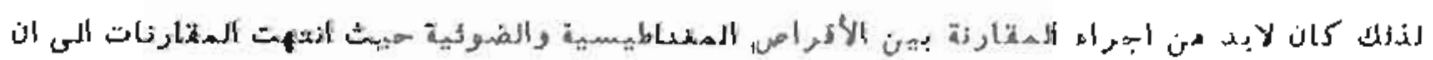

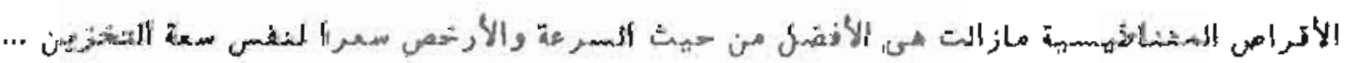

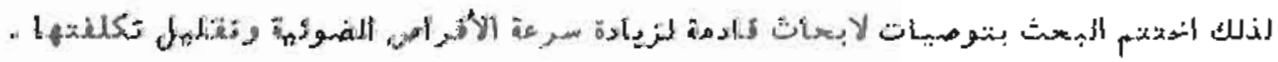




\section{ABSTRACT}

The operating system is a collection of programes (algorithms) designed to manage all the computer system resources, such as processors, main storage and secondary storage, and input/output devices. Optical and magnetic disks are the most common secondary storage devices. The work described in this paper is mainly directed to the optical disk. Different optical disk storage technologies are discussed, in terms of information. about physical disk storage, file systems and access methods. Comparisons are also made between magnetic and optical disk storages.

Keywords: Computer Operating Systems, Disk Scheduling, Optical Technology, CD-ROM, WORM, ODFS.

\section{INTRODUCTYON}

Some application environments such as speech, and graphics require large storage magnitude over that needed for storage text or data alone. Here arises the need for optical storage technology as an alternative to standard magnetic storage (floppy \& hard disks) because magnetic drive is limited by maximum allowable density on the inner track of each disk, due to the geometry of a disk shape makes a track closer to center smaller than one on the outside [12]. Optical disk offers several features, including very high storage capacity, lower cost per bit stored, the ability to remove the disk from the drive, direct access capability and nonerasability. These features make optical disk drives highly desirable for such application as records management, electronic file cabinets for office automation, and network data bases [16]. Write Once optical disks offer a technology that includes very high capacity and removability for handing the information storage requirements of today's [11].

Optical disks bring a new dimension to some applications that have enormous storage capacity, but these are unlikely to replace conventional hard disks. Compact Disk Read only Memory (CD-ROM), Write Once Read Many (WORM), and erasable optical disk technology will let computers perform new tasks and replace such non-computer technology as microfiche. CD-ROM disks are relatively inexpensive to produce and can hold 650 Mbytes each. WORM drives use cartidges and are ideal for archiving because data stored on them can last 10 years. Erasable optical drives are less popular than WORM despite their use in the next computer. WORM and erasable drjves are faster than $\mathrm{CD}$-ROM and become cost effective at capacities of 500 mbytes. 
This paper discusses two different disk storage technologles that are based on the properties of optical material [1]. The first of which stores data by creating permanent changes in optical material of the disk service, and this is used in CD-ROM and WORM. Secondly, the most promising rewritable optical technologies (Erasable optical disk) which uses a laser insted of a magnetic head and stores data in the form of magentic flux. All information about physical disk orgnization, storage, file system and access methods are also discussed $[9,22,24,27]$. Comparisons between magnetic disk storage and optical disk storage are finally given.

\section{OPTICAL DISK DRIVES}

Optical devices employ a read/write head to make physical changes on a rotating medium or disk. The medium is rotated by a spindle which is rotated by a spindle motor. Information is written on the disk as bits of data and these bits are grouped into sectors and tracks [16].

Optical drive like disk which use a controller to convert commands from the host computer into physical actions in the disk drives and to convert parallel data from the host into a serial data stream, which the disk drive expects. Also disk drive control electronics used to convert seek commands from the controller to physical read/write head movements, control the timing of read and write activites and sense conditions in the drive that represent illegal states indicating a fault condition.

As well as Read/Write electronics used to supply the signals necessary to write on the medium and to amplify the signals during read back, so that they can be converted back into digital bits. This can be done by optical unit, which is the heart of the read/write head of the optical disk drive. The goal of this unit is to convey the beam emerging from the laser diode to the underside surface of the optical disk, and then to capture the reflected beam and convey it to a photo detector. The laser beam emerging from laser diode is imperfect. At that point, it is diverging and dissipating rapidly, and it is elliptical in cross section. The beam should be conducted to the underside of the disk so that it makes as small a spot as possible [16].

Emerging from the laser diode, the laser beam goes first through a collection lens. This transforms the laser beam into a collimated (parallel rays) beam. Without step the beam would diverge and dissipate rapidly. Next, the beam passes through prisms, which reshape the beam's cross section from an ellipse to a circle. Then the beam is bounced off a routing mirror to it 90 
degrees, so that it is now headed for the underside of the disk. Finally, the beam passes through an objective lens, which narrows it down to a tiny spot.

After striking the underside surface of the disk, the beam bounces back through the objective lens, which recollimates the beam into parallel rays. At this point, something must be done, so that the beam doesn't retrace its path all the way back to the laser diode. Instead, the beam needs to be channelled to the light detector. This is accomplished by passing the polarized. laser beam through a quarter wave plate, which rotates beam. Now, instead of traveling straight through the beam splitter, as befor, the beam is reflected by 45 degree mirror within the beam splitter, at last being directed to the photo detector.

\section{OPTICAL TECHNOLOGY}

Optical. disk drives use an optics unit with the capability to generate a laser beam. Read/Write head in Optical drives use a high-intensity laser beam to create permanent chanages in a material. These changes on the disk's surface can be read back later as changes in the amount of high reflected to the detector. A single laser is used both for writing and reading. When reading information on the disk, the laser beam is on continuously. The beam strikes the disk and is able to determine whether a particular spot on the disk has a bubble or no bubble (one or zero), the light reflected from a bubble area is greater than that reflected from a non bubble area. When writing, the power level of the semiconductor laser is increased, and instead of the beam being on continuously, it is pulsed on and off in accordance with the on-off sequence of digital information that is being recorded $[16]$.

The information is burned onto the platter in circular tracks. Each track is divided into numerous sectors. The tracks are much closer together than with a magnetic disk. The addressing system for the data is similar to that for magnetic disk. The data have both a track address and a sector address, to make sure that the read/write head is at the correct position, the head and drive stop to read the track and sector address befor executing any write or read command.

Optical disk is a nonerasable media, changes made on the optical disk are permanent. To generate the write beam, most optical disks use a semiconductor laser diode. In order to write, the semiconductor laser is momentarily pulsed to generate 20 or more milliwatts of output power. This power is focused onto the 
recording layer to form permanent deformations. Read back data that have been recorded, the disk must be illuminated. with laser light. This beam can be provided either by the same laser that is used to write or by a separte. Only, when reading, the laser beam is operated continuously and at a much lower power level (1 to 2 miliwatts) than when writing. The recorder information on the tracks of the disk causes the reflected light to be amplitude or phase rodulated. A photodetector then converts these signals into a current that is proportional to the amplitude of the light striking it. This current is finally converted into a voltage that is subsequently decoded to recover the recorded signal.

The laser beam used to read and write can be focused through use of a lens, into a small spot. Since an optically transparent layer can be placed on the media between the read/write head and the recording layer to protect the recording layer. The beam passes up through the disk substrate (transparent glass or plastic), penetrates a thin polmer layer, and strikes the under side of a very thin metal layer. For media derived from video disk technology, the protective layer is a 1.2 millimeter thickness of transparent plastic or glass. The recorded information thereby protected from physical damage. The protective layer also acts as a defocusing layer, making it immune to dust contamination. For an optical system, though, the read/write head can be spaced several millimeters away from the disk. This feature allows the disk to be removed from the drive for safe offline storage.

\section{PHYSICAL DISK ORGANIZATION AND STORAGE}

Physical disk organization is concernd with disk formating and sector addressing. These described in the following subsections.

\subsection{Physical Formating:}

Most magnetic disks use constant angular velocity (CAV) format, where the sectors in the inner tracks are smaller than those on the outer tracks [1]. It is commonly known as a constant rotational speed. The sector organization of typical (CAV) is shown in figure (11). The figure shows that the sector on the outer tracks are physicaliy longer than those on the inner tracks and are organized in concentric rings. All three types of opitcal disks (CD-ROM, WORM and Erasable Optical Disks) use the same techniques for disk organization, but this section focusing on CD-ROM type as it is the most widely used of them. 
The CD-ROM format is based on a constant linear velocity technique (CLV). Where all sectors have the same length, the longer outer tracks contain more sectors. The tracks are spirally organized. This is shown in figure(2). The CLV means that relative speed of the disk head and disk surface stays the same, even the head is moved away from the center of the disk. The CD-ROM drive maintains this CLV by changing the disk's rotational speed as the head moves from track to track. The number of sectors encountered in a signal disk rotation from 9 on the inside of the disk to about 20 on the outer edge [12]. Therefore, recording must be done in a spiral rather than in a series of concentric rings. Recording begins at the inside of the disk and spirals out ward. The general equation governing the motion takes the form: $\quad V=W R$

Where $V=$ linear speed of the disk underneath the optical pickup,

$W=$ the angular speed or the speed of the disk motor, and

$R=$ the distance from the center of the disk.

It can easily be seen that in a CAV system, $W$ is held constant, so the linear speed $V$ must change as $R$ changes. In CLV system, $V$ is held constant so as $R$ changes, the disk motor speed $W$ must change.

The advantage of CAV over CLV format is that the CAV organization makes it easier to find the beginning of a particular sector, where each track contains a fixed number of sectors. It is easy to translate this relative sector number into an absolute track and sector address [12]. In CLV, there is no fixed relation ship between a CLV track and the number of sectors on the track. Random seeking on CD-ROM is quite different on magnetic drives. Essentially, the head is moved near the correct sector in one movement, then is more finely positioned by peeking at the sector passing beneath the head. On magnetic drives the head is moved directly to the correct data to come into position as the disk rotates. While in CLV translating a relative sector number into an absolute track and sector adress is a more complicated process. The motor that spins the disk on a CD-ROM drive must change the angular velocity as the head moves over the disk, drive spins 200 to $500 \mathrm{rpm}$. Compared with magnetic media, this changes in velocity causes seek times and latency times to be relatively high [21].

The advantage of CLV format over CAV is the amount of information that can be stored using CLV. In a CAV system the data on the disk is more dense at the center of the disk than the outer edge. Thus space at the outer edge is wasted. In a CLV system the density of the data on the disk is the same at every point on the disk [21]. Some optical devices have the capability of using either CAV or CLV. 


\subsection{Physical Addressing:}

CD-ROM has inherited some nomenclatures caused by CD digital audio technology. If you use think in terms of playing the disk formated from beginning to end, you find that there is room to record 60 minutes worth of data. Each minute of recording can be divided into 60 second. In each second the driver will read, 75 sector, so the entire disk can hold 270,000 sectors [12].

The sector is the smallest addressable unit on the disk, can be specify in terms of the minute of play, the second within that minute and the sector within that second. One minute of storage on a CD equals 1 minute of playing time which in turns equal 4500 sectors of digital data. The origin of the disk is specified as 0:0:0 (zero minutes, zero second, sector zera). For example the $156^{\prime}$ th sector on the disk is specified as $0: 2: 5$.

\subsection{Disk Capacity and Physical Block Size:}

CD-ROM disks have an extermely large raw capacity. However, over head associated with block formatting, error detection, and error correction requirements cause that capacity to shrink slightly. Many current mastering processes allow only 60 minutes of data on a disk instead of 74 minutes defined by formate [21]. There are two recording modes for $C D-R O M$. Mode 1 is for general data, mode 2 is compatible with the audio data storage formate of compact disks. User data, in mode 1 , is stored on a CD-ROM in 2048 byte blocks. In addition to those bytes, each physical block and for error handling. There are 288 bytes of error correction coding (ECC) data. The ECC segment consists of 4 bytes for error detection, 8 bytes of $0^{\prime} \mathrm{s}$ and 276 bytes of error correction data. At the beginning of each block, 12 bytes are set aside for drive synchronization and 4 bytes for the absolute adaress of the block. After the 2048 bytes of user data, this makes for physical block of 2352 bytes on a CD-ROM. Also mode 2 allows 2336 bytes. Same as mode 1 except that there are no synchronization and absolute address.

\section{OPTICAL STORAGE STANDARDS}

Three types of optical recording technology are available and they are Compact Disk Read Only Memory (CD-ROM), Write Once Read Many (WORM), and Erasable Optical disks. 


\subsection{Compact Disk Read Only Memory (CD-ROM) Optical Disk:}

The advantage of the Compact Disk Read Only Memory (CD-ROM) disk is the high area density which can be recorded. With a linear density of about 25,000 bits per inch (about 25 percent greater than the highest magnetic bit density achievable today), and a track density of about 15,000 (about 10 times the highest achievable today with magnetic recording), net user data density is about 320 mbits per square inch, or about 15 times as high as the hightest-density magnetic disk products available today.

The rotational speed of a typical CD-ROM disk varies between about 200 and 500 revolutions per minute. Access times are on the order of one-half to two seconds, again dictated by the low-cost seek mechanism and servo systems that have become common due to the modest performance requirements of audio applications.

Unlike magnetic disks which are recorded at a constant angular bit density and operate at Constant Angular velocity (CAV), CD-ROM disks have Constant CLV. This permits greater storage capacity than CAV recording because higher area density is achieved at the outer tracks than at the inner ones. On the other hand, rotational speed must be varied from track to track, leading to a more complex servomechanism, CLV disk can hold 50-60 percent more data than one in CAV format, but will pay a penalty in access performance for the increased capacity.

CD-ROM disks are most suitable for electronic publication of information for applications in which large, unchanging database are utilized by many users[1]. They can provide electronic access to large bodies of static information such as:

- Encyclopedias, dictionaries, and catalogs,

- Computer Software,

- Historical data such as financial records, and

- Scientific data such as CRC Tables or Chemical Abstracts.

Although they can make very large amounts of randomly accessible information available at low cost, CD-ROM optical disks require a substantial investment in information prepaxation and master disk development.

Interesting future application possibilities for CD-ROM disks include mixing graphical or audio information with text or other binary data for such application as :

- Computerized navigation; where a database describing a geographic area could be combined with external feedback to provide continuous vehicle position information.

- Computerized catalogue lookup; where textual information about products or services could be combined with video and/or audio representations. 
- Computer-assisted instruction (CAI); where conventional CAI could be augmented with actual video or audio images of the subject matter.

The CD-ROM optical disk is the least expensive device in optical storage disks. It presents the faster means of bulk replication of information. A compact disk containing over 600 Mbytes of formatted user data and it can be replicated in about 15 seconds. The CD-ROM disks can be written only at the factory by specialized equipment and for all suppliers it uses the same protocols and it is readable for all players.

\subsection{Write-Once Read-Many (WORM) Optical Disk:}

Write Once Read Many (WORM) optical disk, as the name implies, can only be meaningfully written once; futher attempts at writting obliterate. WORM technology can be compared in many ways to writing with an indelible pen. A given portion of a WORM disk is written individually, one block at a time by the end user's disk drive, rather than being factory-mastered in large batches.

Areal density of WORM disk is comparable of that of CD-ROM disk, or about an order of magnitude greater than 10 times of magnetic disk. WORM disk drives have areal density comparable to that of CD-ROM drives, but slightly higher transfer performance due to larger typical disk diameters and slightly higher rotational speeds. WORM drives are becoming available in 3.5, $5.25,8,12$ and 14-inch form factors, for a variety of system classes. Rotational speeds of 400-600 revolutions per minute are typical, yielding instantaneous transfer rates in the range of 100-400 kbytes per second[1].

Some WORM disk drives are capable of operating either in CAV or CLV mode. In CAV mode, media capacity is lower, because linear density is lower at the outer diameters. On the other hand since rotational speed is constant, seeking and track centering are similar to magnetic disk applications. In CLV mode, media capacity can be as much as as 50-60 percent greater than of CAV, but access performance is lower, as media speed must change as the drive moves from track. Servocontrol for track centering header analysis cannot begin until rotational speed has been for the new radius and settled to a constant value. Data storage characteristics make WORM disk technology especially attractive for archival application, in which data is saved permanently as a historical record, with no intent of media reuse. It provides a 
new degree of data security for financial, legal, medical, and other application in which the integrity of data contents is of paramount importance.

Because of the archival nature of their applications, data transfer rate is a determining factor in wORM write performance. Factors that can be exploited to improve the performance of WORM disks in the future include

- Rotational speed : which, as with magnetic disks, comblnes with linear density to determine instantaneous data transfer rate.

- Media diameter : which affacts linear velocity past the laser beam.

- Media sensitivity: which determines how long the laser must be applied to a given media spot to alter is reflectivity.

- Laser power : which affects the time required for a laser beam to alter the media's reflectivity.

\subsection{Erasable Optical Disks:}

While CD-ROM and WORM optical technologies are complementary to magnetic recording and will probably result in the development of new applications, erasable optical recording is a direct functional equivalent to magnetic disk recoraing. Erasable optical recording, on the other hand provides random read/write access to individual blocks of data-exactly equivalent to magnetic disk functionality. The differences between erasable optical and magnetic recording technology lie in cost, performance, and removability, rather than function or application. But current erasable implemenation have performance and media constraints, resulting in limited usage in multiuser enviroments[1]. All. erasable optical recording technology are aimed at solving the same technical problem, use of a laser to alter media characteristic to represent data in away that is reversible.

Several different media technology for erasable optical recording have been demonstrated including :

- Magneto-optic recording: in which a laser is used to raise the local media temperatures so magnetic recording can occur. Data is read from magneto-optic media by observing variations in its reflection of polarized light.

- Phase transition recording : in which the laser is used to alter the state of localized areas on the media between amorphous and crystalline, changing its reflectivity. Phase transition recording has the advantage of being read compatible with worm media. Its disadvantage is that media lifetime expressed in number of overwrites may be limited. 
- Photochromic and photodichromic recording : in which the laser is used to change the media color and polarization response properties respectively. These media are sensistive to ambient light, and must therefore be stored in a dark room.

All above current erasable optical technologies require two stages for writing. The first stage, is the erase pass, the laser operates continuously at writing power to bring the media area to be overwritten to a consistent magnetic state. In the second stage, write pass, the laser is modulated by the encoded data pattern. Two-stage writing has the effect of increasing the rotational latency of optical disks, further lowering their performancep+30xrelative to magnetic disks.

Magneto-optic recording provides data densities comparable to the other optical technologies on easy to handle, durable, removable media. A magneto-optic disk with a s-inch diameter typically has storage capacity of about 500 Mbytes. A typical 12-1nch magneto-optic disk might store between 1 and 2 Gbytes.

online read/write access to very large amounts of data, where performance is a secondary consideration to online storage cost, magneto-optic recording is a very promissing technology. Magneto-optical technology is becoming increasingly common in the next few years as :

- Manufacturing processes for drives and media nature.

- Longevity of data recorded is verified.

- The magneto-optic disk industry standarizes on a media technology and recording format.

- Computer system manufacturers integrate erasable optical disk drives into their product lines for purchase by end users.

\section{LOGICAL PORMAT}

Logically, a disk contains a volume table of contents (12), a directory structure and files, physically, the disk contains sectors. The view is the one of interest, the one that determines how many files can store on optical, how large a file can be, and whether files must consist of sequential sectors. As yet there is no agreed upon standard logical format on optical. Any application on optical offer their own logical structure.

There is the lack of industry wide agreement on the relationship between the computer, the CD-ROM drive, the disk, and the data on them. There is not yet any agreement on the way in which the data is organized on the djsk, or on the interface 
between the $C D$ and the rest of the microcomputer world. The result of this lack of agreement is a jungle of competing, nonstandard ways of organizing data on disks, accessing files and conversing with the drive to get what is necessary [9].

Recently, Daniel[24] suggested file management system for a magentic disk used as a buffer to write once optical storage. The design for a magnetic buffer is based on a file management system that uses paging techniques for data transfer between the optical device and the magnetic device. Capacity of the optical media is much greater than that of the magnetic buffer. Use of a magnetic disk as a buffer eliminates the need for major changes to existing operating systems in order to interface with worm optical storage.

File systems were designed to interact with multiple read/write media, they are based on the assumption that once written, an area in storage may be rewritten. Blocks written on WORM optical media may be written once. A multiple read/write buffer eliminates the need for changes to existing file management systems due to this write-once characteristic of secondary storage by intercepting commands from the file management system and handing all direct communication with the optical Disk File Management System (ODFMS) as shown in figure (3). The multiple read/write buffer allows information to be written to the optical device only when the optical disk is physically ejected from the drive, or when the magnetic buffer becomes full and must locate some free space in order to proceed.

Besides eliminating the need for changes to existing operating system, the use of magnetic media as a buffer can minimize the writing of temporary data to the optical disk. The slower access times for optical devices also provides some justification for the use of a multiple read/write magentic disk buffer. The average access for optical drives currentiy exceeds $100 \mathrm{~ms}$ in most cases [24]. For many on-line application, this is an unacceptable feature. The use of high-speed magnetic storage as a buffer can allow portions of a required file to be transferred to the buffer, where faster access times are possible.

\section{OPTICAL DISK FILE SXSTEM (ODFS)}

The major difference between this file system and a typical hierachical file system is that all internal (stored) references to file must be done by fully-qualified pathnames. This is because backward references (reference from one directory to its parent by physical location) would cause extensive searches every time there was any directory unpated. Instead, each directory 
contains its own fully-gualified pathrame and the name of file (or directory) contained it. When a file is to be opened, it always descened the directory tree from the root directory.

An Optical Disk File system (ODSF) is in development at the Advanced Technology of Ricoh Corporation's San Jose, Califomia, factlity [20]. Some of the features of the ODFS are summarized in the following. The ODFS is designed to be interfaced with minimum difficulty to a wide variety of operating systems. The ODFS ts designed in four levels. It minimizes the data copying that would appear to be necessary to keep files and directories up to date. It can be removed at virtualiy any time without lossing the ability to reconstruct the latest state of the file system.

In case of power failure or program error, all files which have been closed and all data which has been written will be recoverable in subsequent use. No auxiliary files on read/write media are required. In case of media failures losses are limited to data in sectors which literally cannot be read. The ODFS writes the latest file data, directory update and file control information in the same area of disk so that seek distance is minimized. This helps to compensate for the inherent low speed of data access.

By implementing variable-length records at the lowest layer (Level 1), the file system allows file aixectories to define variable-sized "extents" naturaliy. This leads to further efficiencies in directory file size. Level 1 implements a volume of vardable length records, so that the sectorfzed structure of the disk is no longer visible. Its records are variable-length and tagged. It uses duifferent calls, such as, Data Read, Data Write, Control Write, Read Record, Write Unmount Record.

At Ievel 2, a Journal, which is sequence of control records is defined. These records contain notes about changes to the file directories. In addition, the File Header records, are written as control records at any time that are changed. This structure is buffered in RAM as weil as on the optical aisk. As new directory changes are made, new journal entries are made in the RAM journal and also written to the disk is minimal, because the control records are small.

Level 3 is concerned with the open and close operation. To search on open file, always descend the tree and cross-check in the journal for any updates to directories, to inspect the journal first. When a file is closed, if it has been modified or extended (or deleted), a new File Header record is written, followed by a journal record indicating the update for the 
directory entry refering to this file. (The airectory entry contains the file name, a reference to the file Header location, and copies of selected information from the File Header.) The journal record is also kept in RAM in a journal Buffer. When it is time to remove the optical Disk Cartridge or to shut down the system, an Unmount operation is performed, This causes the oDFS to examine the journal, update each directory that has been logically modified, and write a new Unmount Record.

Level 4 , is a layer that is intended to permit adaptation of the ODFS for any operating system environment. Data forms adjustments, calling parameter sequences and other apparent incompatibles are to be dealt with in this layer.

\section{ACCESS METHODS}

The two basic access methods, sequential and direct, are available on optical devices. With either approach head movement and latency times can be minimized with proper file layouts on the disk [21].

There are two attributes concerning files are important for the CD-ROM environment, file placement and file structure. An application desiger can and should plan the location of data placed on a CD-ROM disk. Choosing appropriate areas of the disk, and structure for the data are two of the most important design tasks for optimizing CD-ROM application access times. Minimizing access time normally transiate into finding date placement patterns that minimaize head movement and/or latency times.

Optical disk consists of four general file structure that are suited to $C D-R O M$, which are sequential, indexed sequential access method, B-Tree, and Hash $[12,21]$. There are some general rules of thumb that are useful when considering placement of files on CD-ROM:

1- Place data that is frequently accessed together with blocks physically near one another (cluster the data) .

2- Use inner tracks for most frequently accessed data to reduce latency.

3- Place files that are used in conjunction with each other physically near each other on the disk.

4- Interleave files when application will use them concurxently (most appropriate for sequential access files).

5- Avoid situation where the head must move frequently across a large file to get to another file. 
In the case of WORM, there are two methods for storing data. These are sequential and linked access files. Sequential file layout dose not allow files to be updated, but only copied. The directory and the file allocation table notiully found on a disk are simulated in memory as part of the driver. This information is passed to operating system $(0 / \mathrm{S})$ necessary. The simulated disk look just like a convential $0 / S$ disk. Logical to physical translation table is used to convert the logical o/s access information to the physical track and sector references on the laser disk. This conversion is performed for every read or write access. This approach allows any set of files on the worM to be placed in the simulated disk. The sequential nature of the physical disk format lends itself to fast access.

The linked files layout wORM allows files to be updates on a block basis. It also keeps a record of what has been updated by block. The simulated disk information (which is created in memory) is jaentical to the layout of sequential fules, however. the way information is obtained for the logical to physical translation table is aifferent. Aile is initially written as a sequential file with one exception, each block has an additional link which is zero. This can be changed because zeros in the laser medlum can be overwritten. If information has been updated, this link is changed from the old block number to the new block number. The WORM alsk driver is smart enough to know that the logical to physical translation table must be updated and that these links should be followed when building up the initial directory and file allocation tables. The advantage of linked files is that they produce a large updatable medium with $a$ automatic audit trail. The disadvantage is slow sequential access for files that have been updated.

Physical blocks are arranged on CD-ROM tracks in a sequential manner. This differs form magentic media. Magentic disk controllers often formate their media so that physical sectors are interleaved. Interleaving is done when the time between sectors on magentic disks is too short to allow a controller to clear its buffer. By skipping a couple of sectors between sequentially addressed sectors. If interleaving was not used, then a full disk revolution would be requried between sector reads.

CD-ROM transfer speed and the assembly of data from its physical encoding is so slow that automatic interleaving by the controller is not encoding is so slow that automatic interleaving by the permastering phase that allows blocks of files to be interleaving on tracks. One might chooseto do this if the sequence of block accessing is known ahead of time and head 
movement is to be optimized. The provision of interleaving is part of the logical file structure for CD-ROM, not part of the logical file structure for CD-ROM, not part of the physical structure [21].

CD-ROM access time is slow, an average access time very from 500 to 700 . Average latency is quoted at 66 to 70 millisecond for the inner tracks, with 150 milliseconds for the outer tracks. Compared with winchester magnetic technology, with total access times routinely in 13 to $65 \mathrm{millisecond}$ range. Latency times alone for CD-ROM make it a much slower media, without even constdering seek times [12]. It is clear that CD-ROM. Fiqure(4) copmares access times for several devices. It is clear that CD-ROM is much slower than conventional drives or wORM orives.

CD-ROM drives, data is transferred at 176 kbytes/second for the entire physical block of 2352 bytes. However, user data is only 2048 bytes of that total, so the effective transfer rate is more $11 \mathrm{ke} 150$ kbytes/second during a continuous read. This compares with malnframe drives that are on the order of 3000 kbytes/second and personal computer that range from 400 to 1200 kbytes/second (which on average of $800 \mathrm{~kb} / \mathrm{sec}$.). The copmarison is stated in figure(5). It shows that the transfer rate speed of a CD-ROM is the slowest one. This is leads to one the disadvantages of the CD-ROM.

\section{COMPARTSON BETWEEN OPTICAL AND MAGNETIC DISK}

Comparisons between optical and magnetic disk are summarized in table (1) and can be explained in the following.

\subsection{Similarites Between Optical \& Magnetic Disk :}

Both optical and magnetic technologies employ read/write heads to make physical changes on a rotating medium (disk). The medium is rotated by spandle. The spindle is rotated by a spindle motor. Information is written on the disk as bits of data, and these bits are grouped into sectors and tracks.

\subsection{Differences Between Optical \& Magnetic Disk :}

1- Magnetic based on a magnetic head, but optical use optics unit with the capability to generate a lasex beam.

2- Magnetic head creates a high density magnetic field to locally change the orientation of the magnetic domains in iron oxide or plated media. Optical uses a high intensity 
laser beam to create permanent

3- Magnetic drives read/write medium,

changes in material. read many.

4- Magnetic use constant angular velacity (CAV) recording, while Optical base on constant linear velocity (CLV).

5 - Magnetic sectors in the inner tracks are smaller than those on the outer tracks, this causes constant rotational speed. With a CAV format, the linear velocity of the disk surface relative to the disk head is greater on the outer tracks. In the optical disk case, CLV means that the relative speed of the disk head and disk surface stays the same. optical maintains this CLV by actually changing the disks rotational speed as the head moves from track to track. CLV format results in sectors that ald have the same length. This means that the actual number of sectors encountered in a single aisk rotation ranges are vary from the inside of the disk to the outer edge.

6- Maqnetic: Each track contains a fixed number of sectors, it is easy to translate this relative sector number into an absolute track and sector adaress.

optical : There is no simple fixed relationship between a CLV track and the number of sectors on the track. Therefore, translating a relative sector number into an absolute track and sector address is more complicate. Head movement must be accompanied by the mechanical process of speeding up or slowing down the rotational speed of the disk. Together these account for a major part of the CD-ROM's relatively poor seek performance.

7- In Maqnetic, seek time range from 1.6 to $30 \mathrm{~ms}$, but in Optical seek time range from 100 to $500 \mathrm{~ms}$.

\subsection{Advantages Optical Over Magnetic Disk:}

1- The information storage density on laser disk is much higher than on a magnetic. Optical permiling up to 10 times of similar magnetic and low cost per bit stored.

2- An optical disk together with the information stored on it can be mass replcated inexpensively unlike magnetic disk.

3- The laser disk can be removed from its drive allowing the disk itself to be used for archival storage because the read/write head of the optical disk drive is located several millimeters away from the disk but the megantic head disk cannot be removed.

4- Long archival life (more than 10 years versus the two to three years of storage provided by magnetic disks).

5- Direct access capability instead of sequential access in 
magnetic tape.

6- On line secondary storage instead of off line storage magnetic tape. Such information would be more rapidiy accessable than if stored on tape-off time.

7- The read/write head of the optlcal disk is located several millimeters away from the disk, compared to fractions of micrometer in a typlcal magnetic disk drive. The makes optical disks eliminate of head crashes and relatively immune to dust and other contamination. The optical recording process is such that the medium is immune to erasure from external magretic fileds or self exasure.

8- Optical disk will compete favorably with magnetic storage media for applicaltion environments that require storage of such unformatted data types as text, bit-maps, graphics, voice, and programs.

\subsection{Disadvantages of the Optical as Compared to Magnetic Disk:}

1- Complicated error management.

2- It can write only once, since information once written onto the disk cannont be erased.

3- It has an access time (ranges from 100 to $500 \mathrm{~ms}$ ) that is much longer than that of a magnetic disk drive (ranges from 16 to $30 \mathrm{~ms}$ ).

To overcome that drawback, some systems integrators ombine the best of both worlds by integrating winchesters with optical disk drives. In such configurations, the fast access winchester is used for active, or frequently used, files, while the optical drive stores inactive, or infrequently accessed, files [22].

Writing software for the optical drives presents a problem because most off-the-shelf code is in an embryonic stage and is often geared toward magnetic media. Petrocon[22] solves that problem with a mix-and match combination of custom and offthe-shelf optical software that includes its own Al program. The software from Adyanced storage concepts is interface code that manages the optical disk. The program emulates the Dos file structure, which causes a lot of overhead (Min-Micro Systems).

\section{CONCLUSION}

Optical disks bring a new dimension to some application that have enormous storage capacity, but that are unlikely to replace conventional head disk. Compact Disk Read Only Memory (CD-ROM), Write Once Read Many (WORM), and Erasable optical disk technology 
will let computers perform new tasks. So optical disk presents a new area of research in both online and offline seconderay storage systems. Opportunities research try to solve the main draw back of the optical aisks which is access time. Slow access time is the primary negative aspect of CD-ROM that application designers must deal with. The review outlined in this work shows that, optimizing file structures, file accessing algorithms are the best approaches to improve access time performance. Three rules to improve access time are defined. First never access the disk twice, when once is enough. Second never place a file where it slows down overall performance. Third, never structure a file so that multiple accesses are needed to get a single piece of data if it can be structured for one access.

This paper sumarizes two different disk storage technologies, which are based on the properties of optical material. The first of which creates permanent changes in the optical materlal of the disk service, and this used in CD-ROM and WORM. The second, uses a laser beam to store data in the form of magnetic flux. Also, all information about aisk organization, storage, file system, and access method are also explained.

In this work, comparisons are also made between optical and magentic disk on the bases of velocity, number of sector, address, seek time, storage density, access time, and error management. Future possibilities to improve tracking system. Radial tracking system on the optical are not standardized. CD-ROM drive dose not count tracks during the rapid seek mode. This arises out of frequency band contention problems with the data signales. The subsequent fine jumbs can absorb significant time especially as the slow rotational latency compounds the problem. Also there are nonstandard ways of organizing data on disk, accessing files and conversing with the drive to get what is necessary. This means a lot of extra work for developers and a lot of inconvenience for customers.

Future research could involve the design of a file system giving those features: retaining a complete history of all files and directories, selection and retrieval of earlier versions of a file, providing a mulitlevel or hierarchical file system, identifying individual optical disk cartridges, and keeping a master list of all cartridges introduced into the system. 
RTIIIRTNCES

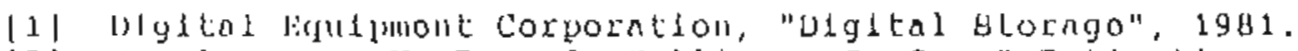

(2) Manolopuos, Y. P. and Koljlas, J. G., "Estimating Disk Head Movement In Batched Searching." BIT (Denmark), VOL. 28, NO. 1, PR. $(27-36) 1988$.

(3) Peterson, J., "Operating System Concepts, "Addison-Wesley, 1985.

(4) Daniel, S. and Geist, R., "A Continual of Disk Scheduling Algorithm," ACM, Vol. 5, 1987.

15) Xiaonan Tan, J. and Towsley, D., "A Performance Analysis of Minimun Laxity and Earliest Deadine Scheduling in a Real time System,"IEEe Trans. on computers, vol, 38, No. 12, Dec. 1989 .

[6] Bel1. $\Lambda$, "Optical Data storage - $\Lambda$ Status Report," in Proc. 6th IEEE Symp. Mass Storage Syst., PP. (93-98), 1984.

(7) Rathman, P." "Dynanic Data Structures On Optical Disks," in Proc. IEEE Data Eng., Conf., PP. (35-42), Nprial, 1984.

(8) Rothchild,E.S.,"An Eye Optical Disks, "Datamation, PP. (73-82), March 1986.

(9) Helliwell, J., "Optical Overview," PC Magazine, PP.(149-164), october $14,1986$.

[10] Seymour, J., "Awaiting The CD-ROM Boom," PC . Magazine, PP. $(87-88)$, May $27,1986$.

(11) Laud, L. "The Evalution of Mass storage, "Byte, PP.(111-118). September 1986

(12) Zoel11ck, B. "CD-ROM Software Development, "Byte, PR. (177-188). September, 1986 .

(13) Dulude, J.R., "The Application Interface of Optical Drtves," Byte, PP (193-200), September, 1986.

(14) Malloy, R.," Mround Up of Optical Disk Drivers," Byte PP. $(73-78)$, September 1986 .

(15) Tim,O., "The CD-ROM Connection."Byte, PP.(315-320), Dec. 1988.

[16] Fujltani. T., "Laser optical disk: the comming revolution $1 \mathrm{n}$ on-line storage," Byte, Vol, 27, PP. (546-554), September, 1986.

(17) Chrlstodulakis, S., "Design And Performance Consideration For An Optical Disk-based, Multimedia Object Server," IEEE Computer Magazlne, PP. (45-56), December, 1986.

(18) Rosch, W. "WORMS For Mass Storage, Based On Laser Beams and Precision", PC Magazine, PP. (135-166), June 23, 1987.

(19) zilber, J., "Optical Horizons", Mac User, Pe.(157-167) June, 1988 .

$\{20\}$ Levy, J. $A$. and Wang, $W ., " \lambda$ File structure For Non-erasable media," IEEE Symposium on mass storage systems, PP. (72-76), Nov. 1988.

(21) Sherman, C."CD-ROM," MCgraw-llill book 1988.

(22) Simpson, D.," Is Write Once The Right Choice," System Integration, PP. $(42-47)$, May 1989.

[23] Weber, S., "Imag1ng: Electrondc Imaglng Systems," Electronlca vol. v62, pp. $(60-65)$, July, 1989.

[24] Canas, D. A. and Bulgren, W.G. "A Flle Management System for $\Lambda$ Magnetic Disk Used $\lambda s$ $\lambda$ Duffer To Write-once optical Disk" The Journal of syster and software, Vol. 10, pp. (15-21).
1989.

(25) Burke, J. J. and Royan, B.," Gigabytes on line," Bytes, PP. (260-266), September 1989.

[26] Gait, J.." A Checkpolnt Pago store For Write-once optical D1sk," ILEE Transaction on computer, Vol. 39, pp. (1-9), January, 1990.

[27] Preview of OE/Boston and OPTCON" 90 spectal events" OE REPORTS", no. 82 , October 1990.

[28] John, R.," Optical Dlsks," Mac User, Vol. v5 issue: no. 11, pp. (132-143), Nov. 1989 .

(29) Charles, S.,"Erasable Optical," Macworld, pp. (152-159), March 1990 .

(30) John, R, "Erasable optical Drives," Mac User, pp. (102-130), November, 1990 . 


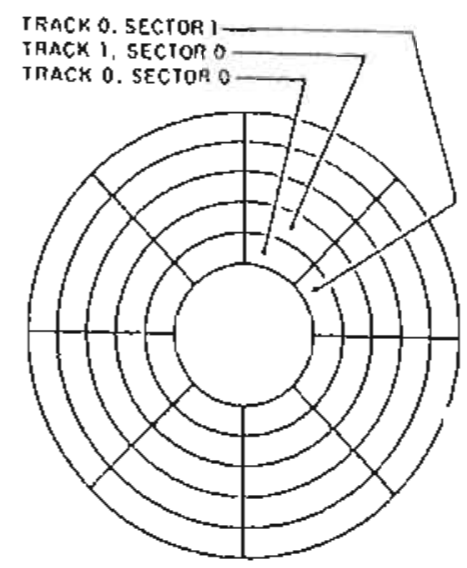

Figure (1): Sector organization of a typical (CAV).

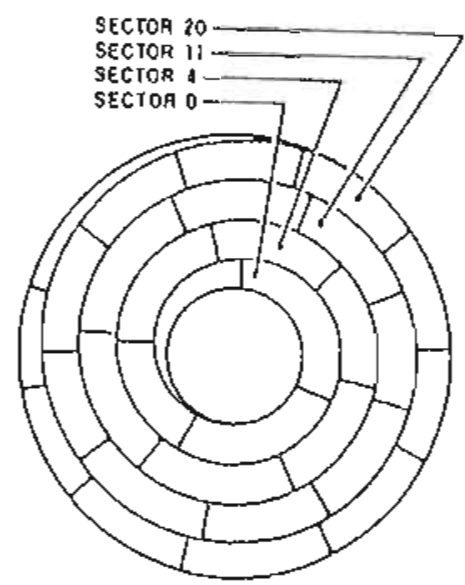

Figure ( 2): seclor organization on a (CLV).

OPERATINO SYSTEM

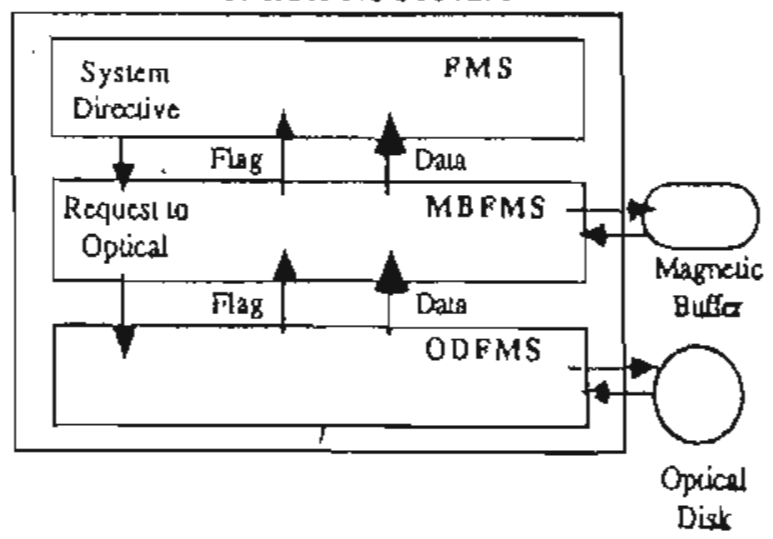

Figure ( 3 ) High speed magentic storage as

a buffer to Optical Disk. 
E.40 A.t.El-Desouky, N.H.Hegazi, M.M.Salem, 0.A.Zahran

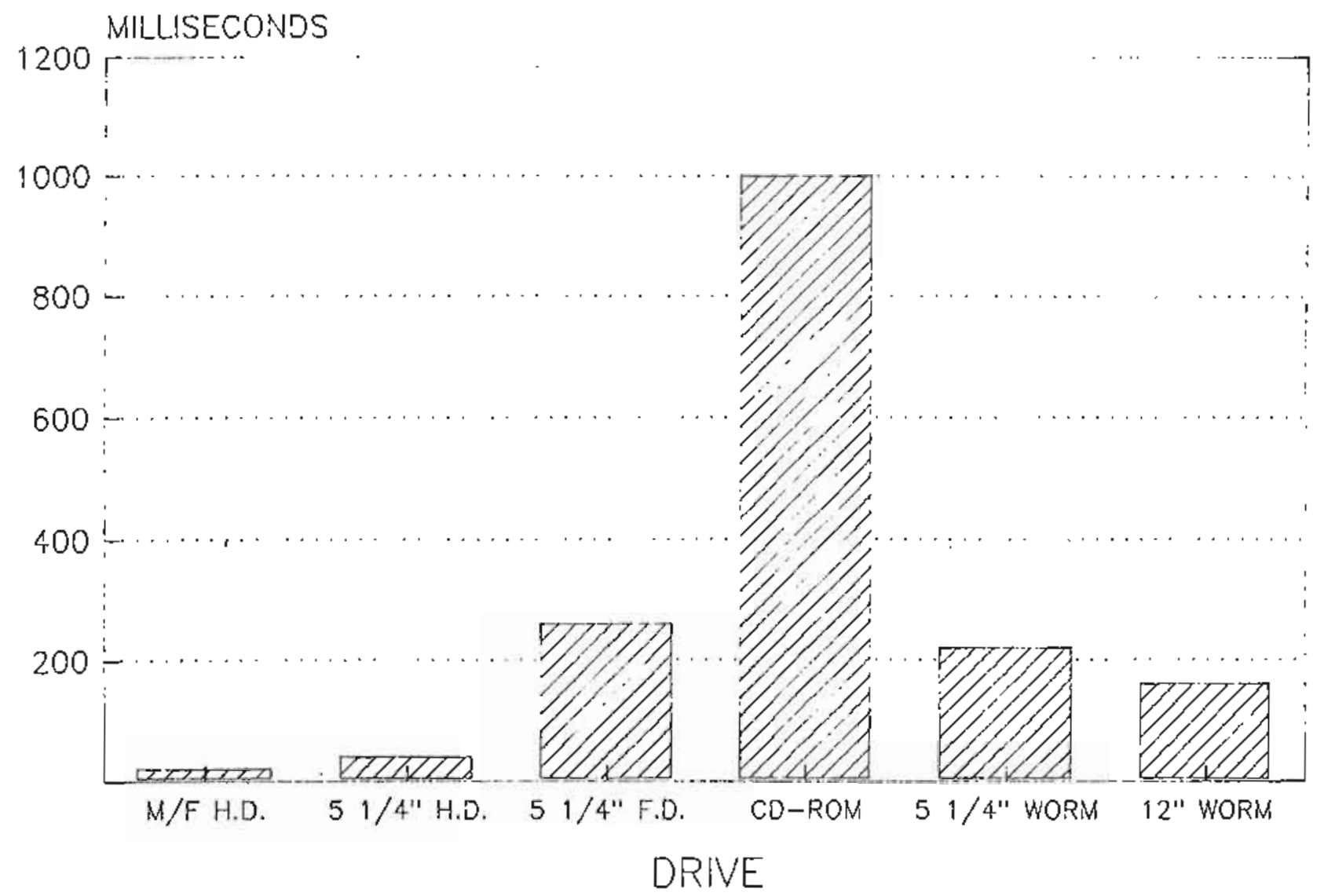

Figure( 4 ) Drive Accoss Speeds (mode 1)

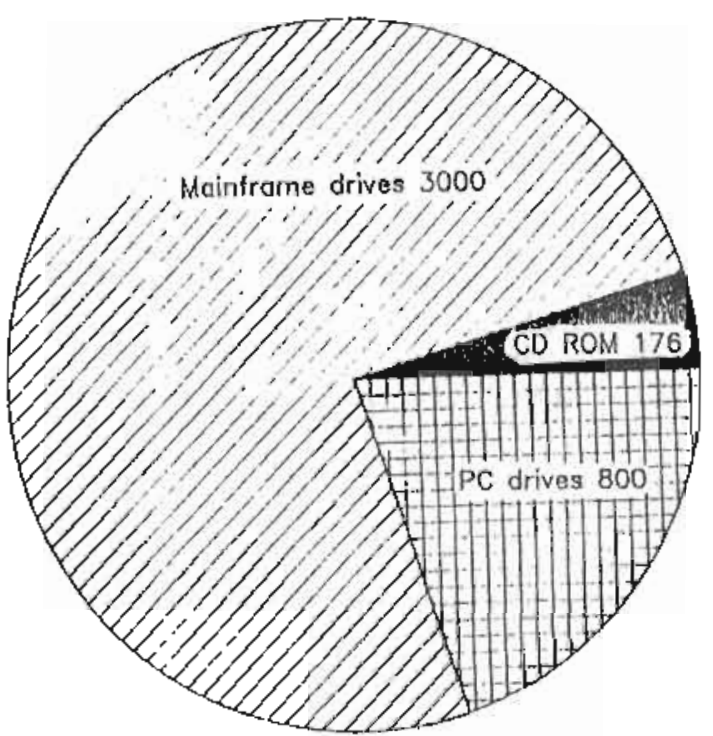

Figure ( 5 ) Drive Transfer Speed 


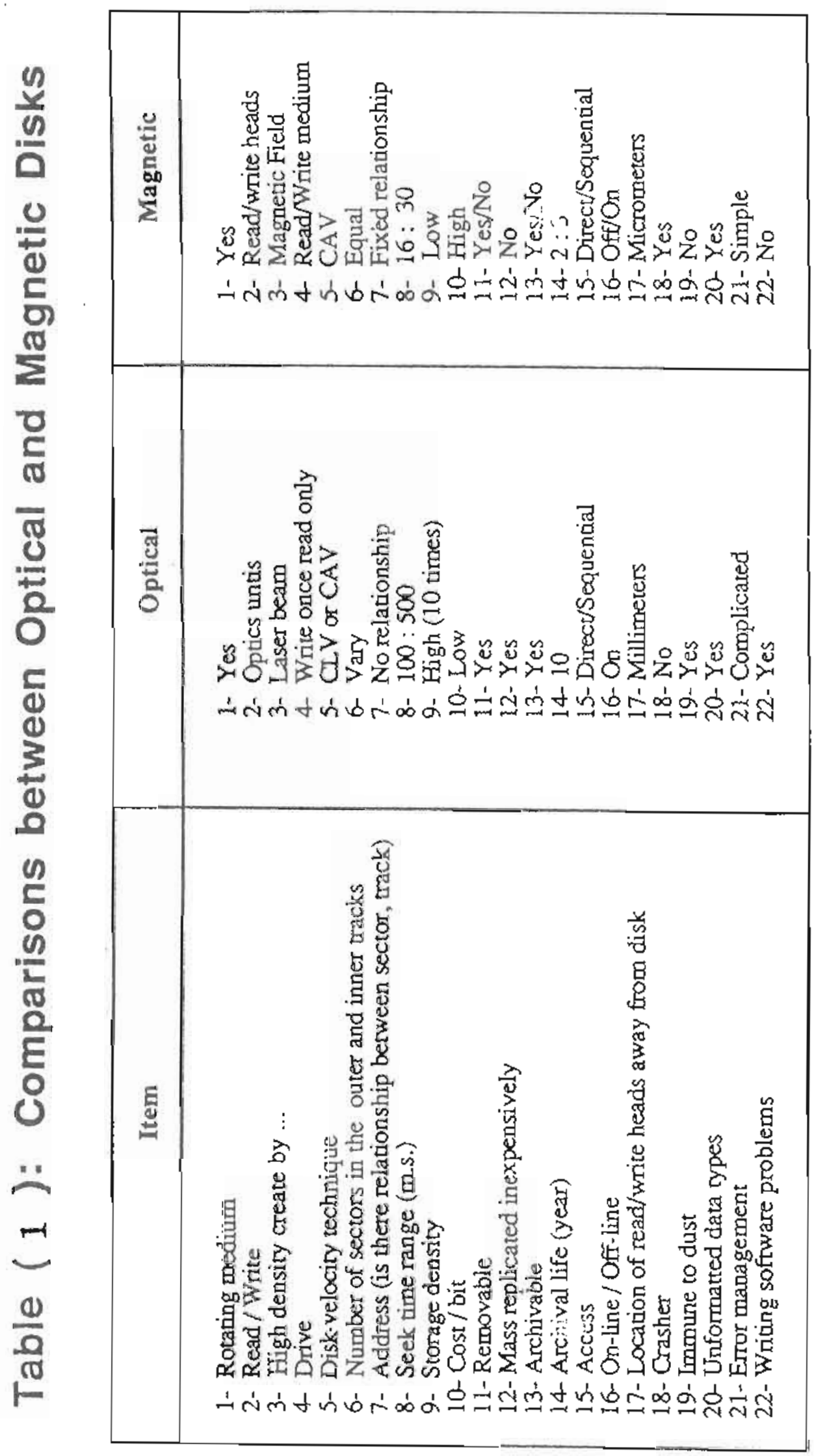

